\title{
Multilocus Variable Number of Tandem Repeat Analysis for molecular typing of uropathogenic Escherichia coli
}

Reza Ranjbar ( $\square$ rezaranjbar@yahoo.com )

Baghiatollah University of Medical Sciences: Baqiyatallah University of Medical Sciences

Farhad Safarpoor Dehkordi

University of Tehran

Morteza Mashhouri

Baqiyatallah University of Medical Sciences

Omid Farahani

Islamic Azad University Varamin-Pishva Branch

\section{Research}

Keywords: Uropathogenic Escherichia coli, Genotyping, MLVA, VNTR

Posted Date: October 2nd, 2020

DOI: https://doi.org/10.21203/rs.3.rs-84882/v1

License: (c) (i) This work is licensed under a Creative Commons Attribution 4.0 International License.

Read Full License 


\section{Abstract}

Background: The aim of this study was genotyping of Uropathogenic Escherichia coli (UPEC) based on Variable Number of Tandem Repeats (VNTRs) sequences.

Methods: $E$. coli strains isolated from urine samples were included in this study. Seven VNTR loci were subjected to Multilocus variable-number tandem repeat analysis (MLVA) based on PCR amplification. Then data was analyzed via online mlvaplus software and the information was displayed in the form of MST analysis.

Results: A total of 100 E. coli strains were isolated and subjected to the study. MLVA was able to differentiate 56 different genotypes. Also, the technique could classify $E$. coli isolates in 5 clonal complexes. Based on UPGMA dendrograms, $E$. coli isolates were classified into 4 clusters (clusters A to D). The strains associated with Complex No. 1 appeared to be dominant pathogens of UPEC in Tehran's patients. The present study provides valuable insights into the genetic relationships of $E$. coli isolates recovered from clinical cases in a major hospital in Iran.

Conclusions: The analysis of MLVA profiles using the MST algorithm showed the usefulness of the MLVA method in the classification of uropathogenic $E$. coli collected in different periods. We evaluated MLVA in a laboratory equipped with simple molecular equipment. Based on these results, it has been assumed that the $E$. coli strains were derived from a limited number of clones that have undergo a small genetic change during this period.

\section{Background}

Escherichia coli (E. coli), a flagellated member of Enterobacteriaceae, is an essential member of the normal flora in the human gut, but many kinds of symptoms are caused by various $E$. coli pathotypes with a wide range of virulence factors. For instance, septicemia causing $E$. coli (SCEC) and neonatal meningitis producing E. coli (NMEC) are ExPEC pathotypes as the main cause of septicemia and pediatric meningitis respectively. Another important member of EXPEC pathotypes is uropathogenic E. coli (UPEC) which causes urinary tract infections (UTIs) [1-6].

There are several methods for typing of $E$. coli from environmental samples including traditional and modern molecular methods [7-13]. With the advent of the molecular age, scientists changed their approach from phenotyping to genotyping methods $[14,15]$.

Different molecular techniques such as Repetitive element sequence-based PCR (rep-PCR), Random Amplified Polymorphic DNA (RAPD)-PCR, Ribotyping, Pulsed-field gel electrophoresis (PFGE), and Multiple Locus Variable-number Tandem Repeat Analysis (MLVA) have been used for genotyping of different strains of $E$. coli up to now. Among them, the MLVA method has been proposed as a new molecular method for bacterial genotyping $[16,17]$. In MLVA technique, only a PCR-based method is required, while other sophisticated method such as PFGE need specialized and expensive equipment's. In 
this way, MLVA is a low-cost alternative to PFGE, especially in developing countries [18]. It can also be used in simple laboratories that are only equipped with a PCR machine [19]. In this study, we tried to design a new, fast, and low-cost method for genotyping of uropathogenic $E$. coli by choosing suitable VNTR repeat sequences.

\section{Methods}

\section{Sample collection and E. coli isolation}

Over a 12-month sampling period (from October 2018 to October 2019), E. coli isolates were recovered from patients with UTI in a major hospital in Tehran, Iran and subjected to the current study. Isolation of the E. coli was done by conventional standard biochemical and serological methods. Well-isolated colonies of purified $E$. coli were resuspended in trypticase soy broth with $20 \%$ glycerol and stored in $70{ }^{\circ} \mathrm{C}$ for long-term storage [8].

\section{DNA preparation}

A pure culture of $E$. coli was plated on nutrient agar (Merck, Germany) and incubated overnight at $37^{\circ} \mathrm{C}$. A single colony was removed from the plate, suspended in $200 \mu \mathrm{l}$ of sterile deionized water, and boiled for $15 \mathrm{~min}$. After centrifugation at $6,000 \mathrm{~g}$ for $8 \mathrm{~min}$, the supernatant was transferred into a new tube for subsequent PCR analysis [20]. Purity (A260/A280) and concentration of extracted DNA were then checked (NanoDrop, Thermo Scientific, Waltham, MA, USA). The truth of the DNA was assessed on a $2 \%$ agarose gel stained with ethidium bromide $(0.5 \mu \mathrm{g} / \mathrm{mL})$ (Thermo Fisher Scientific, St. Leon-Rot, Germany).

\section{MLVA assay}

For genotyping, using the MLVA technique, seven loci VNTRs were used based on the protocol of O Gorgé, S Lopez, V Hilaire, O Lisanti, V Ramisse and G Vergnaud [21]. These seven VNTR loci were: ms06, ms07, ms09, ms11, ms21, ms23 and ms32. The primer sets for PCR amplification of these VNTR loci were previously reported by O Gorgé, S Lopez, V Hilaire, O Lisanti, V Ramisse and G Vergnaud [21] (Table 1). PCR was performed in $25 \mu$ volume including $1 X \mathrm{PCR}$ buffer $(50 \mathrm{mmol} / \mathrm{L} \mathrm{KCl}, 10 \mathrm{mmol} / \mathrm{L}$ Tris, $\mathrm{pH}=9$ ), $2.5 \mathrm{mmol} / \mathrm{L} \mathrm{MgCl} 2,0.2 \mathrm{mmol} / \mathrm{L}$ of each primer with $1 \mathrm{U}$ of TaqDNA polymerase (CinnaGen Co., Iran), and $4 \mu \mathrm{l}$ of the crude DNA extract. 
Table 1

MLVA primers and annealing temperatures for PCR reactions.

\begin{tabular}{|c|c|c|c|c|}
\hline $\begin{array}{l}\text { MLVA } \\
\text { locus }\end{array}$ & Forward primer $5 \square$ to $3 \square$ & Reverse primer $5 \square$ to $3 \square$ & bp & $\begin{array}{l}\text { Annealing } \\
\text { temperature } \\
\left({ }^{\circ} \mathrm{C}\right)\end{array}$ \\
\hline $\mathrm{ms06}$ & AAACGGGAGAGCCGGTTATT & TGTTGGTACAACGGCTCCTG & 39 & \multirow[t]{7}{*}{55} \\
\hline $\mathrm{ms} 07$ & GTCAGTTCGCCCAGACACAG & CGGTGTCAGCAAATCCAGAG & 39 & \\
\hline $\mathrm{ms} 09$ & GTGCCATCGGGCAAAATTAG & CCGATAAGGGAGCAGGCTAGT & 179 & \\
\hline ms11 & GAAACAGGCCCAGGCTACAC & CTGGCGCTGGTTATGGGTAT & 96 & \\
\hline ms21 & GCTGATGGCGAAGGAGAAGA & GGGAGTATGCGGTCAAAAGC & 141 & \\
\hline $\mathrm{ms} 23$ & GCTCCGCTGATTGACTCCTT & CGGTTGCTCGACCACTAACA & 375 & \\
\hline ms32 & TGAGATTGCCGAAGTGTTGC & AACTGGCGGCGTTTATCAAG & 101 & \\
\hline
\end{tabular}

We introduced the number of replicates for each locus in each isolate, along with other characteristics of the isolates into Microsoft Excel, and then put these data on the WWW.mlvaplus.net site. The frequency of VNTR loci repeats is entirely different from each other by electrophoresis on the agarose gel. MST is a convenient complementary tool to cluster multiple isolates and visualize the relative diversity within different lineages.

A dendrogram of genetic relationships was also generated using the unweighted pair group method with arithmetic averages (UPGMA) method [22]. Furthermore, Simpson's index of diversity (D) and 95\% confidence intervals $(\mathrm{Cl})$ for each VNTR locus were calculated using version 2.0 phyloviz software (http://www.phyloviz.net). For dendrogram analysis, the UPGMA method and Dice similarity coefficient were used to analyze VNTR data $[23,24]$.

\section{Results}

\section{MLVA analysis}

One-hundred E. coli strains were identified, of these, 70 isolates were belonged to the patients with complete biographical information and subjected to MLVA. Figure 1 describes the polymorphism of 3 VNTR loci in different $E$. coli isolates. As the result of PCR, the DNA band size distributions of the $70 E$. coli strains for each VNTR were 301-379 bp (ms06), 392-821 bp (ms07), 445-1432 bp (ms09), 718958 bp (ms11), 233-1492 bp (ms21), 584-959 bp (ms23), and 355-810 bp (ms32). Simpson's index of diversity, confidence Interval 95\%, number of different alleles per locus and Tandem repeat (TR) size have been counted (Table 2). 
Table 2

VNTR locus, tandem repeat size, Simpson's index of diversity and confidence interval $95 \%$.

\begin{tabular}{|llllll|}
\hline $\begin{array}{l}\text { VNTR } \\
\text { locus }\end{array}$ & $\begin{array}{l}\text { TR size } \\
\text { (bp) }\end{array}$ & $\begin{array}{l}\text { Simpson's index of } \\
\text { diversity }\end{array}$ & $\begin{array}{l}\text { confidence } \\
\text { Interval95\% }\end{array}$ & No. of alleles & $\begin{array}{l}\text { Null } \\
\text { alleles }\end{array}$ \\
\hline ms06 & 39 & 0.6301 & $0.587,0.6732$ & $2(3,4)$ & yes \\
\hline ms07 & 39 & 0.5921 & $0.498,0.6862$ & $3(7,8,9)$ & No \\
\hline ms09 & 179 & 0.8066 & $0.7453,0.868$ & $5(2,2.5,5,1,4)$ & Yes \\
\hline ms11 & 96 & 0.681 & $0.6234,0.7387$ & $3(5,4,6)$ & no \\
\hline ms 32 & 101 & 0.7038 & $0.6315,0.7761$ & $7(1,1.5,2,4,4.5$, & No \\
\hline ms 23 & 375 & 0.2532 & $0.1271,0.3792$ & $2(1,2)$ & no \\
\hline ms 21 & 141 & 0.8073 & $0.747,0.8676$ & $3(5,7,4)$ & no \\
\hline
\end{tabular}

\section{VNTR analysis}

The analysis of the number of VNTR repetitions using the MST algorithm showed that in 70 isolates of $E$. coli, 56 different genotypes were observed. However, due to the high similarity of the profile of isolated $E$. coli with each other and also for the better separation of complex colonies, the criterion of difference in a locus for grouping isolates (multiple clones) was considered. Accordingly, E. coli strains were classified in 5 clonal complexes.

\section{Evolutionary relationship between isolates}

Each complex group represents the evolutionary relationship between isolates. In other words, isolates within each group have a genetic correlation that is closer to each other and is likely to have the same origin. In our study, Complex No. 1 was the largest clonal group (Fig. 2). E. coli isolates were classified in 4 clusters (A cluster to D) based on the similarity (Fig. 3).

\section{Discussion}

In this study, we successfully applied the MLVA method to analyze uropathogenic E. coli strains isolated from clinical samples.

MLVA method does not require a high level of expertise and is capable to provide reasonable results about monitoring of outbreaks and clonal spread of bacterial isolates over a short period of time. It has been successfully applied to investigate the clonal relationship and epidemiology of clinical $E$. coli isolates $[21,25,26]$. In this study, the MLVA technique was able to detect 56 different genotypes among 70 E. coli strains isolated from patients with UTI in a major hospital in Tehran, Iran, indicating a high diversity of UPEC isolates genotypes. 
Also, MLVA technique could classify the stains in 5 clonal complexes. In examining UPGMA dendrograms, uropathogenic $E$. coli isolates were classified according to the similarity in 4 clusters (clusters A to D). Each complex group represents an evolutionary relationship between the isolates. In other words, isolates belonging to each group have a genetic association with each other and are likely to have the same origin. In our study, Complex No. 1 was the largest clonal group. The strains associated with Complex No. 1 appeared to be the dominant pathogens of UPEC in the hospital under study.

In the present study, we used markers that can be easily separated by electrophoresis on the agarose gel. On the other hand, the evolution rate of different VNTRs differentiated the clonal relationships. Typically, loops that have larger replication sizes are slower than circuits that have smaller duplicate sizes. But loci with a slower evolutionary velocity are suitable for the investigation of clonal relationships between isolates that have evolved over a more extended period. Therefore, the type of VNTR locus is also in the analysis $[27,28]$.

The applied technique could classify $E$. coli isolates in 5 clonal complexes. Each complex group represents the evolutionary relationship between isolates. In other words, isolates belonging to each group have a genetic link with each other and are likely to have the same origin. In our study, Complex No. 1 was the largest clonal group. The strains in Complex No. 1 appeared to be the prevalent strains of UPEC disease, with about two-thirds of the population receiving all strains.

The ms 32 has the highest number of alleles. The ms 06 and ms23 loci have the smallest amount of alleles, and the ms06, ms09, ms21, and ms23 loci have null alleles. In the dendrogram analysis, The UPGMA of uropathogenic $E$. coli isolates is classified according to the similarity in 4 clusters (clusters A to $D$ ). We also found that to better evaluate VNTR loci for the study of epidemics or genotyping, more and more numbers of $E$. coli are needed. Besides, the use of a higher number of VNTR loci will be more beneficial for better differentiation of clonal groups.

Mellor et al. used the MLVA method to determine the genetic diversity of E. coli 0157 producing Shiga toxin venom. There was some evidence that $E$. coli 0157 strains collected from different regions may also show genetically diversity. To study the amount of this variation, Shiga toxin bacteriophage insertion sites (SBI), lineage-specific polymorphisms (LSPA-6), multilocus variable-number tandem repeat analysis (MLVA), and a tir 255T > A polymorphism were used to enquire 606 isolates from Australian and U.S. cattle and human. All analyses of collected data showed a significant connection between the source of origin and multilocus genotypes $(P<0.0001)$ and it confirms the evolution of enterohemorrhagic $E$. coli 0157 in Australia and the United States [29].

MLVA was also used by Bustamante et al. for subtyping of 202 STEC isolates from different sources to get information about the genetic diversity of native STEC in Argentina. Two different MLVA protocols were used in their investigation, one for 0157 and the other for a generic E. coli assay. As a result of their studies, MLVA was mentioned as a very sensitive tool to STEC subtyping and it showed the diversity in many serotypes [30]. 
Naseer et al. used the MLVA and multi-locus sequence typing (MLST) for subtyping of a total of $100 \mathrm{E}$. coli isolates from Spain, UK, Sweden, and Norway ( $n=19,20,24$ and 37 respectively) and the obtained data showed equivalence between the MLST profile and MLVA typing results [31].

In a study in Norway by Sekse et al, on 142 E. coli 026 isolated from 491 fecal, PFGE and MLVA was performed for investigation of the genetic relationship between the strains and 63 different PFGE profiles and 22 MLVA profiles were identified. Despite some differences in result between MLVA and PFGE, comparison by partition mapping showed good overall agreement between two methods. A few isolates of EPEC 026: $\mathrm{H} 11$ had a close relationship to STEC 026: $\mathrm{H} 11$ [32].

VNTRs regions are widely used for bacterial subtyping but it should not be overlooked that the hyper variability in VNTR loci can be problematic in trying to predict the relationships between isolates. It is also worth noting that knowing the rate of VNTR mutation and its effective factors can introduce the MLVA for legal epidemiological or microbiological research [33].

In a study in Japan on a total of 57 isolates from patients, 20 types were identified by using MLVA while they were classified into 23 PFGE types and the results were almost the same. In another study in the same city, a total of 24 isolates from different sources and one outbreak occurred in central parts of the city was investigated by MLVA an PFGE. The results showed a good correlation between these both methods. However, the MLVA typing proved to be a much more comfortable and more rapid method for the analysis of E. coli 0157: $\mathrm{H} 7$ strain relatedness to identify transmission paths. Hence, MLVA method was appeared to be a convenient method for typical typing in many laboratories, including public health agencies and even in hospitals [33-36].

\section{Conclusions}

The study population consisted of E. coli strains isolated from patients with UTI in a major Hospital in Tehran. The analysis of MLVA profiles using the MST algorithm showed the usefulness of the MLVA method in the classification of uropathogenic E. coli collected in different periods. We also found that MLVA technique was a fast, easy and low-cost method for genotyping of uropathogenic $E$. coli. We evaluated this technique in a laboratory equipped with simple molecular equipment. The present study provides valuable insights into the genetic relationships of $E$. coli isolates recovered from clinical cases in a major hospital in Tehran, Iran. Based on these results, it has been assumed that the uropathogenic $E$. coli strains were derived from a limited number of clones that have undergo a small genetic change during this period.

\section{Abbreviations}

E. coli: Escherichia coli; UPEC: Uropathogenic Escherichia coli, VNTRs: Variable Number of Tandem Repeats; MLVA: Multilocus variable-number tandem repeat analysis; UTIs: urinary tract infections; UPGMA: unweighted pair group method with arithmetic averages 


\section{Declarations}

\section{Ethics approval and consent to participate}

The present investigation was confirmed by the ethical supervision of Scientific Research of the Baqiyatallah University of Medical Sciences, Tehran, Iran. Sampling licenses were issued by Prof. Reza Ranjbar (Ref Number 3607614).

\section{Consent for publication}

There was no consent for publication.

\section{Availability of data and materials}

All data generated or analyzed throughout this research are included in this published article.

\section{Competing interests}

The authors declare that they have no competing interests

\section{Funding}

Not applicable.

\section{Authors' contributions}

RR and FSD designed the study and carried out the PCR genetic alignment and typing. MM and OF supported the study and carried out the samples collection, bacterial isolation and statistical analysis. RR carried out the writing and drafting of the manuscript. All authors road and approved the final manuscript.

\section{Acknowledgments}

Thanks to guidance and advice from Clinical Research Development Unit of Baqiyatallah Hospital, Tehran, Iran.

\section{References}

1. Ranjbar R, Farahani O: The Prevalence of Virulence Genes and Virulotypes of Escherichia coli Strains Isolated from Hospital Wastewaters in Tehran, Iran. Iranian J Public Health 2018;47:713.

2. Ranjbar R, Seyf A, Dehkordi FS, Chen T, Tang W, Chen Y, Zhang J, Xie X, Etemadi A, Moniri R: Prevalence of Antibiotic Resistance and Distribution of Virulence Factors in the Shiga Toxigenic Escherichia coli Recovered from Hospital Food. Jundishapur J Microbiol 2019;12:e82659. 
3. Ranjbar R, Dehkordi FS, Shahreza MHS, Rahimi E: Prevalence, identification of virulence factors, Oserogroups and antibiotic resistance properties of Shiga-toxin producing Escherichia coli strains isolated from raw milk and traditional dairy products. Antimicrob Resist Infect Control 2018;7:53.

4. Hemmatinezhad B, Khamesipour F, Mohammadi M, Safarpoor Dehkordi F, Mashak Z: Microbiological Investigation of O-Serogroups, Virulence Factors and Antimicrobial Resistance Properties of Shiga Toxin-Producing E scherichia Coli Isolated from Ostrich, Turkey Quail Meats. Journal of Food Safety 2015;35:491-500.

5. Dehkordi FS, Yahaghi E, Darian EK: Prevalence of Antibiotic Resistance in Escherichia coli Isolated from Poultry Meat Supply in Isfahan. Iran J Med Microbiol: Volume 2014;8:41-7.

6. Safarpoor Dehkordi F, Yazdani F, Mozafari J, Valizadeh Y: Virulence factors, serogroups and antimicrobial resistance properties of Escherichia coli strains in fermented dairy products. BMC Res Notes 2014;7:217.

7. Ranjbar R, Sami M: Genetic Investigation of beta-lactam associated antibiotic resistance among Escherichia coli strains isolated from water sources. Open Microbiol J 2017;11:203.

8. Momtaz H, Karimian A, Madani M, Dehkordi FS, Ranjbar R, Sarshar M, Souod N: Uropathogenic Escherichia coli in Iran: serogroup distributions, virulence factors and antimicrobial resistance properties. Ann Clin Microbiol Antimicrob 2013;12:8.

9. Jahandeh N, Ranjbar R, Behzadi P, Behzadi E: Uropathogenic Escherichia coli virulence genes: invaluable approaches for designing DNA microarray probes. Central European J Urol 2015;68:452.

10. Ranjbar R, Haghi AM, Joneydi JN, Abedini M: The prevalence and antimicrobial susceptibility of bacterial uropathogens isolated from pediatric patients. Iranian J Public Health 2009;38:134-8.

11. Ranjbar R, Karami A, Farshad S, Giammanco GM, Mammina C: Typing methods used in the molecular epidemiology of microbial pathogens: a how-to guide. New Microbiol 2014;37:1-15.

12. Ranjbar R, Farahani 0 : The Prevalence of plasmid-mediated quinolone resistance genes in Escherichia coli isolated from hospital wastewater sources in Tehran, Iran. Iranian J Public Health 2017;46:1285.

13. Anvarinejad M, Farshad S, Ranjbar R, Giammanco G, Alborzi A, Japoni A: Genotypic analysis of E. coli strains isolated from patients with cystitis and pyelonephritis. Iranian Red Crescent Med J 2012;14:408.

14. Chang AC, Liu BH: Identification of characteristic macromolecules of Escherichia coli genotypes by atomic force microscope Nanoscale Mechanical Mapping. Nanoscale Res Letter 2018;13:1-6.

15. Nakamura $H$, Iguchi $A$, Maehara T, Fujiwara K, Fujiwara A, Ogasawara J: Comparison of three molecular subtyping methods among 0157 and non-0157 Shiga toxin-producing Escherichia coli isolates from Japanese cattle. Japanese J Infect Dis 2017;71:45-50.

16. Ranjbar R, Mostafavi Shokouhi S, Memariani H: Evaluation of multiple-locus variable-number tandem repeat analysis (MLVA) for genotyping of Escherichia coli isolated from Karaj River. Water Science and Technology: Water Supply 2018;18:160-6. 
17. Helldal L, Karami N, Welinder-Olsson C, Moore ER, Åhren C: Evaluation of MLVA for epidemiological typing and outbreak detection of ESBL-producing Escherichia coli in Sweden. BMC Microbiol 2017;17:8.

18. Arya G, Holtslander R, Robertson J, Yoshida C, Harris J, Parmley J, Nichani A, Johnson R, Poppe C: Epidemiology, pathogenesis, genoserotyping, antimicrobial resistance, and prevention and control of non-typhoidal Salmonella serovars. Current Clin Microbiol Report 2017;4:43-53.

19. Sánchez GR: Identification and typing methods for the study of bacterial infections: a brief review and mycobacterial as case of study. Arch Clin Microbiol 2015;7:1-10.

20. Ranjbar R, Memariani M, Memariani H: Diversity of variable number tandem repeat loci in Shigella species isolated from pediatric patients. Int J Molecul Cell Med 2015;4:174-81.

21. Gorgé O, Lopez S, Hilaire V, Lisanti O, Ramisse V, Vergnaud G: Selection and validation of a multilocus variable-number tandem-repeat analysis panel for typing Shigella spp. J Clin Microbiol 2008;46:1026-36.

22. Ranjbar R, Memariani $H$, Sorouri R, Memariani M: Distribution of virulence genes and genotyping of CTX-M-15-producing Klebsiella pneumoniae isolated from patients with community-acquired urinary tract infection (CA-UTI). Microb Pathogen 2016;100:244-9.

23. Casarez EA, Pillai SD, Di Giovanni GD: Genotype diversity of Escherichia coli isolates in natural waters determined by PFGE and ERIC-PCR. Water Res 2007;41:3643-8.

24. Christiansson M, Melin S, Matussek A, Löfgren S, Söderman J: MLVA is a valuable tool in epidemiological investigations of Escherichia coli and for disclosing multiple carriage. Scandinavian J Infect Dis 2011;43:579-86.

25. Memariani M, Peerayeh SN, Salehi TZ, Mostafavi SKS: Occurrence of SHV, TEM and CTX-M $\beta-$ lactamase genes among enteropathogenic Escherichia coli strains isolated from children with diarrhea. Jundishapur J Microbiol 2015;8:e15620.

26. Dormanesh B, Dehkordi FS, Hosseini S, Momtaz H, Mirnejad R, Hoseini MJ, Yahaghi E, Tarhriz V, Darian EK: Virulence factors and o-serogroups profiles of uropathogenic Escherichia coli isolated from Iranian pediatric patients. Iranian Red Crescent Med J 2014;16:e14627.

27. Flatt T, Heyland A: Mechanisms of life history evolution: the genetics and physiology of life history traits and trade-offs: OUP Oxford: 2011.

28. Liao J-C, Li C-C, Chiou C-S: Use of a multilocus variable-number tandem repeat analysis method for molecular subtyping and phylogenetic analysis of Neisseria meningitidis isolates. BMC Microbiol 2006;6:1-11.

29. Mellor GE, Besser TE, Davis MA, Beavis B, Jung W, Smith HV, Jennison AV, Doyle CJ, Chandry PS, Gobius KS: Multilocus genotype analysis of Escherichia coli 0157 isolates from Australia and the United States provides evidence of geographic divergence. App Env Microbiol 2013;79:5050-8.

30. Bustamante AV, Sanso AM, Parma AE, Lucchesi PMA: Subtyping of STEC by MLVA in Argentina. Front Cell Infect Microbiol 2012;2:1-4. 
31. Naseer U, Olsson-Liljequist BE, Woodford N, Dhanji H, Canton R, Sundsfjord A, Lindstedt B-A: Multilocus variable number of tandem repeat analysis for rapid and accurate typing of virulent multidrug resistant Escherichia coli clones. Plos one 2012;7:e41232.

32. Sekse C, Sunde M, Lindstedt B-A, Hopp P, Bruheim T, Cudjoe K, Kvitle B, Urdahl AM: Potentially human-pathogenic Escherichia coli 026 in Norwegian sheep flocks. App Env Microbiol 2011;77:494958.

33. Bustamante AV, Sanso AM, Segura D, Parma AE, Lucchesi PMA: Dynamic of mutational events in variable number tandem repeats of Escherichia coli 0157: H7. BioMed Res Int 2013;2013:1-10.

34. Kawamori F, Hiroi M, Harada T, Ohata K, Sugiyama K, Masuda T, Ohashi N: Molecular typing of Japanese Escherichia coli 0157: $\mathrm{H} 7$ isolates from clinical specimens by multilocus variable-number tandem repeat analysis and PFGE. J Med Microbiol 2008;57:58-63.

35. Murphy M, Minihan D, Buckley JF, O'Mahony M, Whyte P, Fanning S: Multiple-locus variable number of tandem repeat analysis (MLVA) of Irish verocytotoxigenic Escherichia coli0157 from feedlot cattle: uncovering strain dissemination routes. BMC Vet Res 2008;4:1-9.

36. Keys C, Kemper S, Keim P: Highly diverse variable number tandem repeat loci in the E. coli 0157: H7 and 055: H7 genomes for high-resolution molecular typing. J App Microbiol 2005;98:928-40.

\section{Figures}

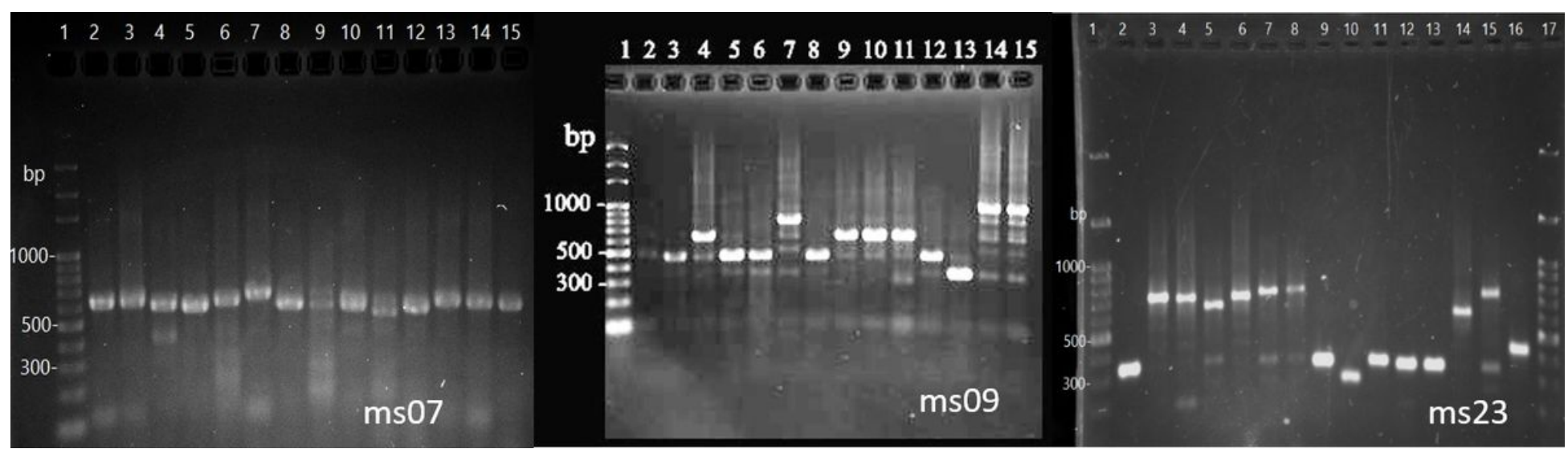

\section{Figure 1}

Polymorphism of 3 VNTR loci in different E. coli isolates. This image illustrates how the number of repeats can be directly deduced by manual reading. 


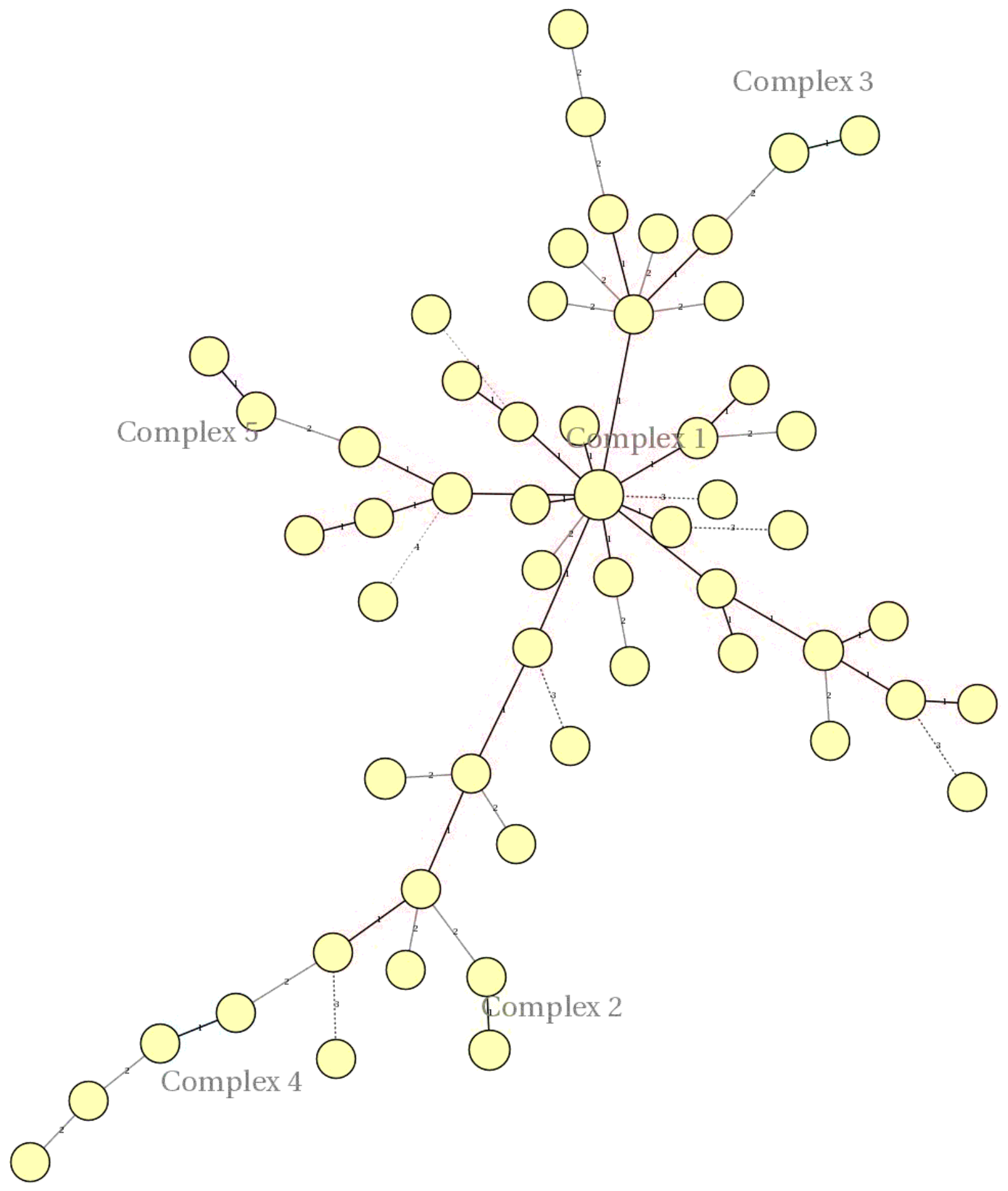

Figure 2

Data analysis by using the Minimum Spanning Tree (MST) algorithm with a difference of 2 loci. each circle is showing an isolate with a unique profile. 


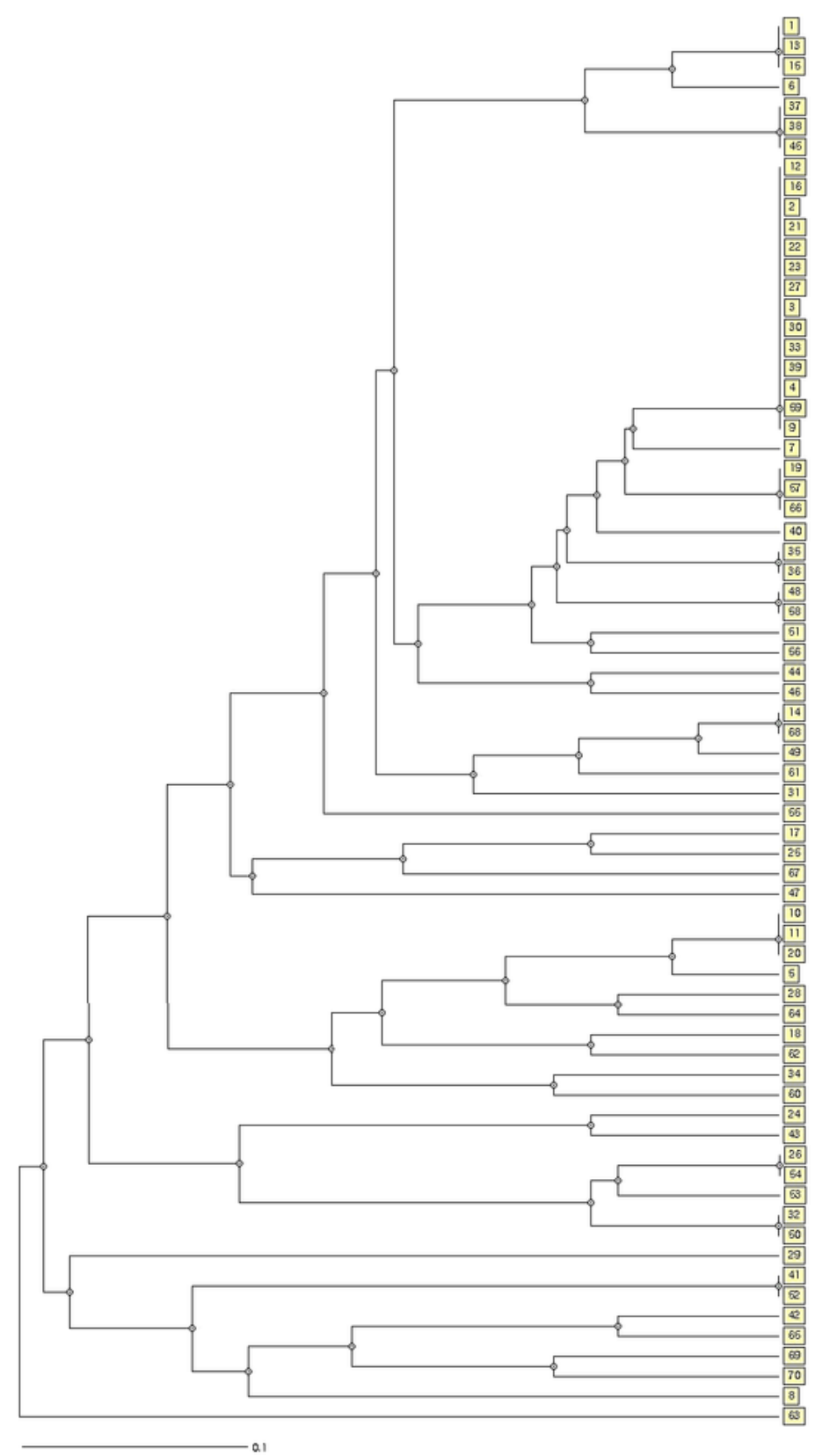

Figure 3

Clustering of the MLVA profiles by UPGMA with the categorical coefficient of similarity. 